\title{
Video Article \\ The Vermicelli and Capellini Handling Tests: Simple quantitative measures of dexterous forepaw function in rats and mice
}

\author{
Kelly A. Tennant ${ }^{1}$, Aaron L. Asay ${ }^{2}$, Rachel P. Allred ${ }^{3}$, Angela R. Ozburn ${ }^{4}$, Jeffrey A. Kleim ${ }^{5}$, Theresa A. Jones ${ }^{1,2}$ \\ ${ }^{1}$ Institute for Neuroscience, University of Texas at Austin \\ ${ }^{2}$ Department of Psychology, University of Texas at Austin \\ ${ }^{3}$ Department of Neurology, University of Florida \\ ${ }^{4}$ Department of Psychiatry, University of Texas Southwestern Medical Center \\ ${ }^{5}$ Department of Neuroscience, McKnight Brain Institute, University of Florida
}

Correspondence to: Theresa A. Jones at tj@psy.utexas.edu

URL: https://www.jove.com/video/2076

DOI: doi:10.3791/2076

Keywords: JoVE Neuroscience, Issue 41, manual dexterity, food handling, pasta, rodent models, upper extremity impairment

Date Published: 7/21/2010

Citation: Tennant, K.A., Asay, A.L., Allred, R.P., Ozburn, A.R., Kleim, J.A., Jones, T.A. The Vermicelli and Capellini Handling Tests: Simple quantitative measures of dexterous forepaw function in rats and mice. J. Vis. Exp. (41), e2076, doi:10.3791/2076 (2010).

\section{Abstract}

Previous characterizations of rodent eating behavior have revealed that they use coordinated forepaw movements to manipulate food pieces. We have extended upon this work to develop a simple quantitative measure of forepaw dexterity that is sensitive to lateralized impairments and agedependent changes. Rodents learn skillful forepaw and digit movements to manage thin pasta pieces, which they eagerly consume. We have previously described methods for quantifying vermicelli handling in rats and showed that the measures are very sensitive to forelimb impairments resulting from unilateral ischemic lesions, middle cerebral artery occlusions and unilateral striatal dopamine depletion [Allred, R.P., Adkins, D.L., Woodlee, M.T., Husbands, L.C., Maldonado M.A., Kane, J.R., Schallert, T. \& Jones, T.A. The Vermicelli Handling Test: a simple quantitative measure of dexterous forepaw function in rats. J. Neurosci. Methods 170, 229-244 (2008)]. Here we present a more detailed protocol for this test in rats and compare it with a newly developed version for mice, the Capellini Handling Test. Rats and mice are videotaped while handling short lengths of uncooked vermicelli or capellini pasta, respectively, with a camera positioned to optimize the view of paw movements. Slow motion video playback allows for the identification of forepaw adjustments, defined as any distinct removal and replacement of the paw, or of any number of digits, on the pasta piece after eating commences. Forepaw adjustments per piece are averaged over trials per each testing session. Repeated testing permits sensitive quantitative analysis of changes in forepaw dexterity over time. Protocols for pre-testing habituation and handling practice, as well as procedures for characterizing atypical handling patterns, are described. Because rats and mice perform the pasta handling tests slightly differently, species-specific differences in administration and scoring of these tests are highlighted. All animal use was in accordance with protocols approved by the University of Texas at Austin Animal Care and Use Committee.

\section{Video Link}

The video component of this article can be found at https://www.jove.com/video/2076/

\section{Protocol}

\section{Preparing for the onset of testing}

1. Uncooked pasta pieces are cut to length using a razor blade. For rats, Skinner brand vermicelli pasta $(1.5 \mathrm{~mm}$ diameter) is cut to $7 \mathrm{~cm}$ lengths. For mice, DeCecco brand capellini pasta (1.0 mm diameter) is cut to $2.6 \mathrm{~cm}$ lengths (Fig. $1 \mathrm{C})$. Because pasta brands vary in texture and diameter, which could influence handling manner, it is recommended that a single source of pasta be used for repeated testing.

2. To overcome neophobic responses and to establish skill in handling, animals are exposed to the pasta pieces in their home cage several times in the weeks prior to testing. (Typically, 5-10 days of exposure, about 4 pieces each time, is sufficient.)

3. If the test will be administered in a chamber other than the home cage, or if the home cage is moved for testing, habituation to eating in the testing environment is provided. (It typically takes 3-5 sessions until animals are readily eating the pasta in this environment.)

4. Even after animals eagerly accept pasta, they can suddenly become reticent when a large camera is moved next to the testing area. It is helpful to place the camera in front of the testing environment for one or more of the habituation sessions.

5. Because most animals act as if the pasta is highly palatable, it is not essential to food restrict them prior to testing. However, removal of food a few hours before or overnight may facilitate eating in front of the experimenter and camera and ensure that they are not sated at the test time.

6. Breaking the pasta piece during eating invalidates a trial. We have found this habit is largely avoided if animals are never given pieces longer than the ones used in the tests. 
7. For the testing sessions, pasta pieces are marked with an ultrafine tip marker at specific intervals using a cardstock template $(1.75 \mathrm{~cm}$ increments on vermicelli and $0.86 \mathrm{~cm}$ increments on capellini strands). This facilitates visualization of the movement of the pasta strand during eating.

\section{Test set up}

1. Animals should be tested in either the home cage or another apparatus with unmarred, clear walls, and should be isolated from their cagemates during the test session.

2. Though rats can be tested in real time by a practiced experimenter (Allred et al., 2008), quantification is facilitated by video recording so that data can be collected during slow-motion video playback. We have not found it feasible to reliably quantify mouse forepaw movements without slow motion video playback due to the small size of their paws and fast movements.

3. A video camera with a high frame rate and adequate zoom is used. We use a Canon XL1S camera with a 16X zoom lens set to a shutter speed of at least $420 \mathrm{~s}-1$ in normal room lighting conditions. The use of a wheeled tripod with a panning and tilting head facilitates camera positioning.

4. Rats typically sit upright on their haunches to eat pasta and the paws are most easily viewed with the camera positioned at approximately chamber floor level with a slight upward angle (Fig. 1A). If testing in a cage with shavings, removal of some shavings may be useful to optimize the view.

5. Mice tend to bend forward during pasta eating, holding the piece at more of an angle than rats do. Thus, a testing chamber with an open floor is placed on top of a mirror. The camera is placed at a slightly higher level than the floor, and angled downward to focus on the reflection of the paws in the mirror (Fig 1B). The use of a first surface mirror greatly improves the clarity of the paws for video playback.

6. Each bite of the pasta piece is audible and, thus, audio recording is useful for verifying eating.

\section{Administering pasta handling trials}

1. Test sessions consist of sequential trials, with one pasta piece eaten per trial. We typically record data from 4-5 trials per rat and 3-4 trials per mouse.

2. Prior to videotaping, a piece of pasta is placed into the chamber to initiate eating and to move the animal into an appropriate camera view.

3. It is necessary for the animal to face the camera and to focus and zoom sufficiently $(\sim 10-12 X)$ to view discrete adjustments with both forepaws and all digits. The knuckles of both paws should be viewable. At least with this camera, we find it useful to use autofocus features only prior to trial onset, to narrow in on the region of interest. We subsequently switch to manual focus.

4. As soon as the animal and camera are in position, the first trial is initiated. A piece is dropped or dangled directly in front of the animal and recording is initiated prior to eating onset.

5. Thereafter, the trials are given in immediate succession. It is advisable to minimize delays between trials so that animals do not move away from the optimal viewing position.

6. Trials in which the paws are viewed to the side or in which focus is lost are not scored.

7. The test sessions can be repeated over days, e.g., to document pre- and post-treatment temporal patterns (e.g., recovery after CNS injury; Allred et al., 2008).

8. A challenge can be getting animals to eat in desired positions relative to the camera. It may be necessary to habituate camera-shy animals to the presence of the camera for a few minutes prior to testing. Animals can often be coaxed into the position by dropping or dangling pasta in the ideal region. It may require a few pieces to accomplish this desired position, though the number of pieces given should be minimized to avoid satiety.

9. For sequential testing of several rats, we have found it convenient to configure a few of their cages at a time in a circle, with the camera on a wheeled tripod in the middle. A piece is dropped into an ideal region of each cage and the camera then moved to the rat that begins eating in an appropriate position first. This allows more time for the slower (to eat in position) rats to become habituated to the camera and experimenter presence and they may also be stimulated to eat by the biting sounds of the faster rats.

\section{Quantitative video analysis of forepaw adjustments and eating time}

1. The analysis of forepaw adjustments during eating is a simple quantitative measure that is sensitive to lateralized impairments after CNS injury in rats (Allred et al., 2008; Whishaw \& Coles, 1996; Whishaw et al., 1997) and to aging-related changes in mice (Tennant et al., 2009).

2. The quantified portion of the trial begins when the pasta piece is first placed into the mouth and ends when the last of the pasta is released from the paws and disappears into the mouth.

3. Video playback is slowed ( $50 \%$ of real-time), or frame-by-frame advance is used, as needed, to identify adjustments.

4. An adjustment is counted each time the paw releases and re-contacts the pasta piece, when there is reformation of the paw hold on the piece, or when there is an extension-flexion or abduction-adduction of the digits (Fig. 2). Each adjustment is recorded separately for the left and right paw.

5. Adjustments are not counted when the paw slides across the pasta piece with no movement of the digits or repositioning of the paw hold. Movements of the paw when it is not in contact with the pasta piece (i.e., movements next to the piece) are also not counted.

6. The time to eat is also recorded for each trial, starting with eating onset and ending when the pasta disappears into the mouth. We record time using video playback display features, but it can also be recorded with a stopwatch during normal speed playback.

7. If the animal pauses in eating, but maintains a hold on the piece, the time paused is subtracted from the total time. A pause in eating is evident by either movement of the piece out of the mouth or a cessation in audible biting.

8. The trial is omitted from the analysis if during any part of the eating, the view of the paws is obscured, or if the animal breaks the piece, because the shorter pieces alter handling patterns.

9. Some rats and mice consistently make more adjustments with one paw than the other. For unilateral CNS injury models, lateralized impairments are more sensitively detected if the injuries affect the paw that made more adjustments in pre-injury testing (Allred et al., 2008) 
10. Mice sometimes drop the pasta piece in the middle of eating, and, after a short break, pick it up and continue eating. This is most common in the last trial of the day, possibly due to partial satiety and loss of focus on eating. Because this is common even in intact mice, we do not omit these trials. However, the time that the piece is dropped is subtracted from the total eating time.

11. In intact rats, dropping pieces is atypical and resumption in eating is often very delayed or absent. Such trials are omitted from the analysis of adjustments and eating time.

12. Adjustments prior to the eating onset are not counted. Pre-eating handling behaviors are affected by CNS injuries (e.g., Whishaw et al., 1992; Whishaw et al., 1986), but we have not yet extended the quantitative methods for forepaw adjustments to this period.

13. These data can be summarized as: (a) the average number of adjustments per paw per piece, (b) the average eating time per piece and (c) the rate of adjustments (adjustments/eating time).

\section{Video analysis of atypical handling patterns}

1. Atypical handling patterns can distinguish between different CNS injury types in rats (Allred et al., 2008) and recording these behaviors may be useful as a supplemental analysis.

2. Handling patterns are considered atypical if they are infrequently observed in intact animals. Intact animals typically initiate eating with one paw, the "grasp paw", placed lower on the pasta, and the "guide paw" is then placed near the mouth (Fig. 3A,C). The same paw is usually the grasp paw in all trials. Rats often position paws far apart along the length of the pasta at first, and move them together as the piece shortens. Mice typically position both paws close to the mouth, with the grasp and guide limbs held close to one another, even at the beginning of eating. In both species, the paws move into more symmetrical positions, side-by-side or with digits interposed, when the piece is very short (Fig. 3B,D). Both paws are in use continuously for guiding, holding or advancing the pasta during eating.

3. Atypical patterns in paw position in rats and mice include: (a.) placing the paws together, in a symmetrical hold, when the pasta piece is long (more than half the original length), (b.) failing to move the paws into a symmetrical position before the piece is completely consumed and (c.) switching positions of the guide and grasp limbs in the midst of eating.

4. Two additional atypical behaviors in rats and mice are: (a.) failing to contact the pasta piece with one paw for an extended time during eating ("failure to contact") and (b.) using the mouth to pull the pasta through the paws to reposition them ("mouth pulling").

5. Two behaviors are considered atypical for rats, but are typical for mice: (a.) hunched posture and (b.) dropping the pasta piece after eating begins. However, the frequency of drops in mice may increase after CNS damage. Thus, we record drop frequency in both species.

6. Other atypical behaviors have been seen in a rat unilateral striatal dopamine depletion model: (a.) an "iron grip", where the grasp limb is held in an unmoving, rigid position during eating, (b.) repeated movement of the guide paw around the rigid grasp paw, and (c.) and extreme tilting of the head as the pasta is angled into the mouth (Allred et al., 2008; see also Whishaw et al. 1997).

7. We have observed that aged mice often make adjustments that are ineffective for moving the pasta into the mouth. Thus, adjustments that do not result in a visible movement of the pasta piece into the mouth are recorded separately.

8. Switching the guide and grasp limbs, failures to contact, drops, and mouth pulling are recorded per occurrence, while all other atypical behaviors are recorded once per trial.

\section{Representative Results}

The results from the video analysis should include the number of adjustments of each paw per trial and the time to eat each pasta piece. Results from rats with sensorimotor cortical lesions or cerebral ischemia indicate that animals make fewer adjustments with the impaired (contra-tolesion) limb, an effect that endures for at least a month after the injuries. Unilateral dopamine depletion results in both a decrease in adjustments with the contralateral limb and an increase in adjustments with the ipsilateral limb (Allred et al., 2008). Results from mice show that aged animals take longer to eat the pasta pieces, and make more adjustments that do not result in advancement of the pasta into the mouth, compared to young adult mice (Tennant et al., 2009). The analysis of these tests may also include observations of atypical handling behaviors. Striatal damage and middle cerebral artery occlusion additionally resulted in increased frequency of abnormal eating behaviors (Allred et al., 2008). 


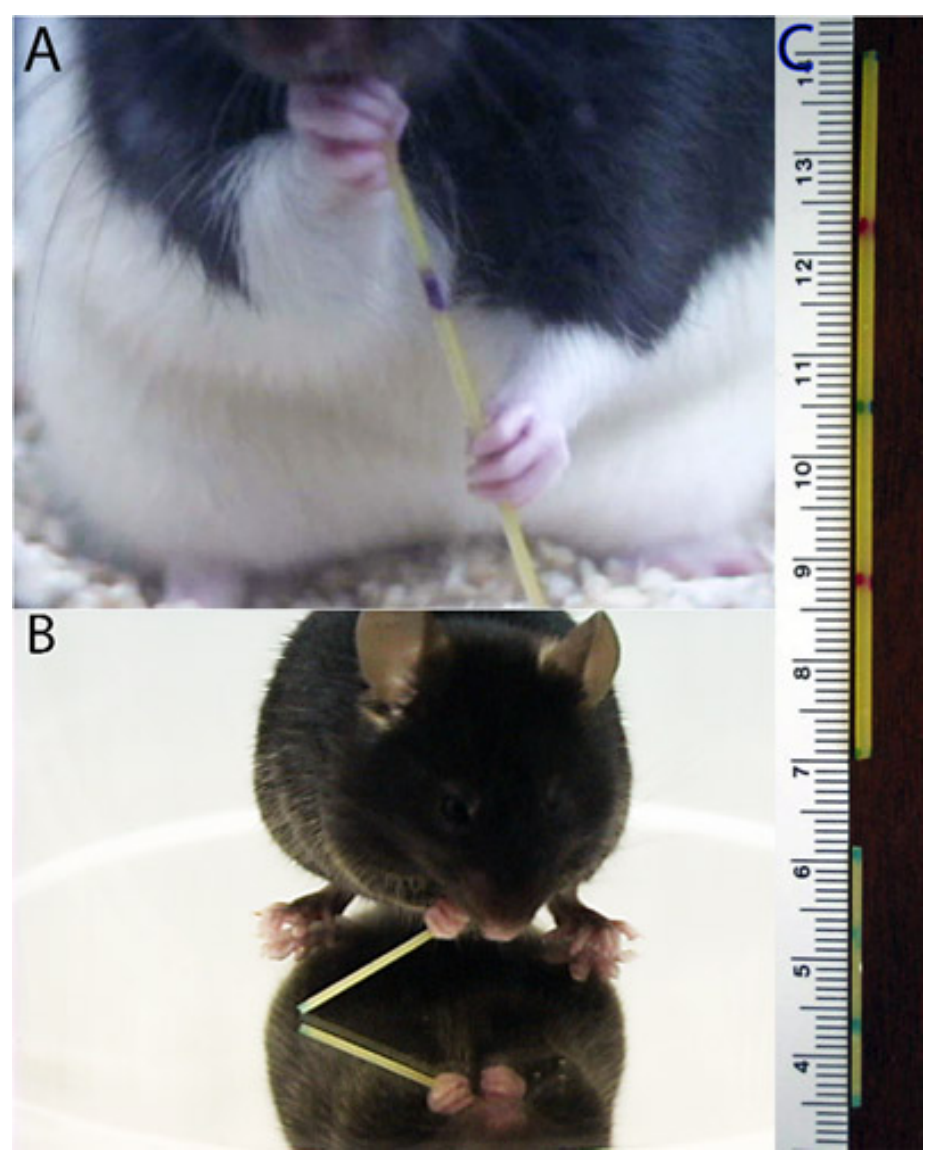

Figure 1. Pasta handling posture of (A) rats and (B) mice. Note that the rats sit back on their haunches while the mice hunch over the pasta, necessitating the placement of a mirror below the mouse in order to view paw movements. (C) Examples of pasta pieces prepared for testing.

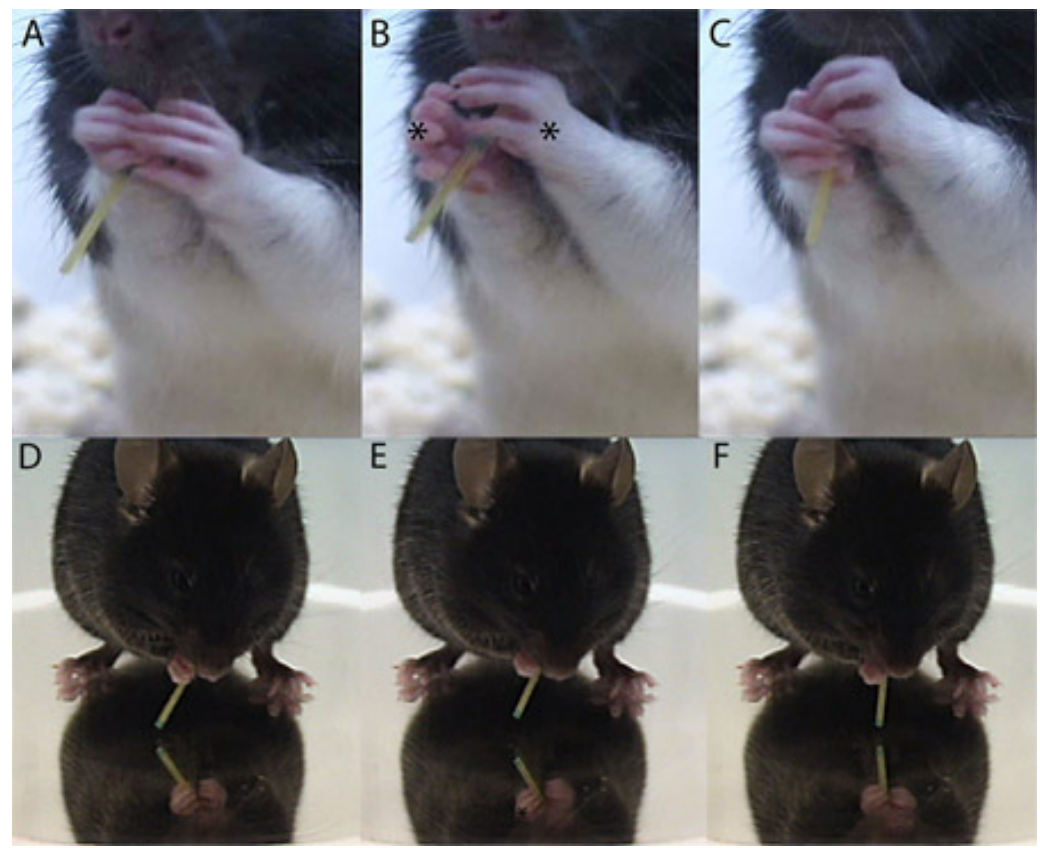

Figure 2. An example forepaw adjustment of $(A-C)$ a rat and $(D-F)$ a mouse. The first panel shows the animal before the adjustment, the center panel shows the forepaw adjusting, with an asterisk indicating the adjusting paw(s), and the last panel shows the replacement on the pasta piece. 


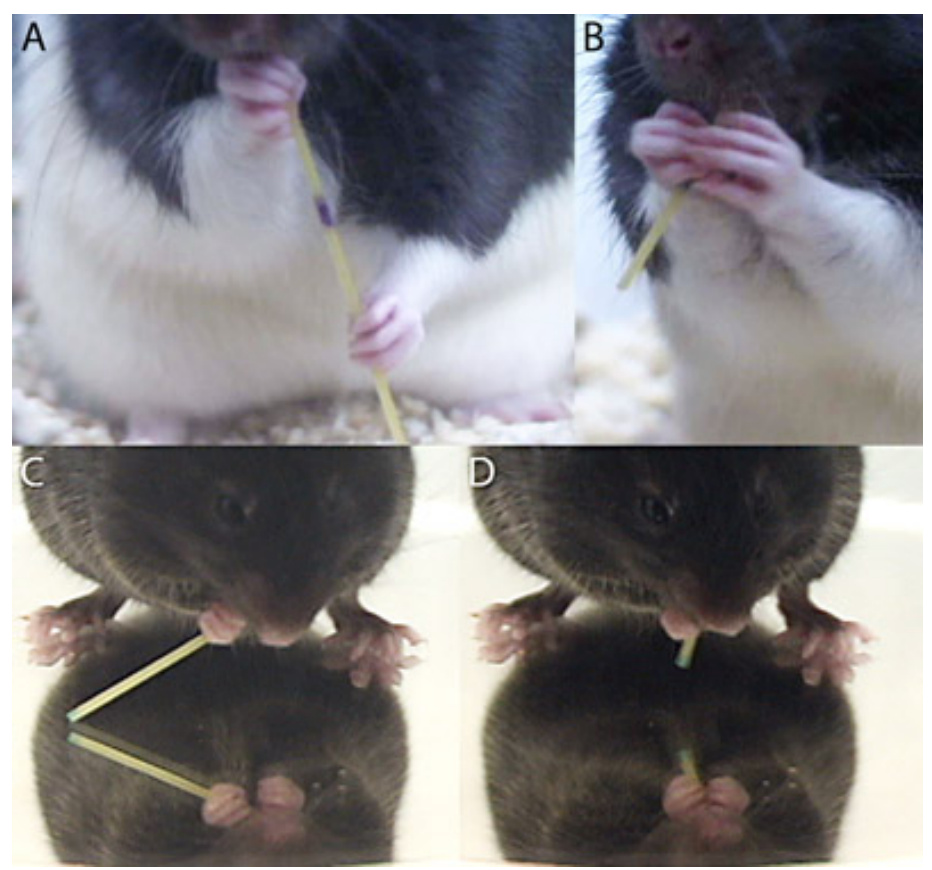

Figure 3. Guide and grasp limb positions of rats and mice when the pasta is $(A, C)$ long and $(B, D)$ short.

\section{Discussion}

In this video, we demonstrate how The Vermicelli and Capellini Handling Tests are conducted in rats and mice, respectively. These tests are a simple, quantitative way to study paw and digit function in normal animals, after damage to motor systems of the brain, or during aging. Slowmotion video analysis of the paw and digit movements used to handle small pieces of uncooked pasta allow the researcher to quantify the adjustments made with each paw, as well as observe abnormal behaviors that may be seen following experimentally-induced CNS damage. Testing sessions can be done repeatedly on different days and most animals complete all trials of a session within a few minutes. Thus, the test is suitable for incorporation into a larger test battery or experimental design. The tests can also be used in studies of motor skill learning (Xu et al., 2009), but here we present a protocol to assay changes in an established skill over time.

Currently, skilled reaching tests are the most commonly used tests of dexterous forelimb function in rats (e.g., Whishaw et al., 1986; Ballermann et al., 2001; Montoya et al., 1991) and mice (Farr \& Whishaw, 2002; Tennant \& Jones, 2009; Baird et al., 2001). Reaching tests permit isolated testing of each limb, whereas pasta handling is a bimanual task. Nevertheless, the tests are similar in their sensitivity to impairments in skilled forepaw function and the acquisition of skill for both tests involves plasticity in the forelimb representation of the motor cortex (Xu et al., 2009). In our experience, the reaching tests are more complicated and laborious to perform and, for some experimental questions, the present tests may provide a more efficient alternative. However, unlike the tests described here, skilled reaching tests have been in use for decades (Peterson, 1934) and they now include a versatile set of highly sensitive measures and test parameters (Ballermann et al., 2001; Farr \& Whishaw, 2002; Montoya et al., 1991; Whishaw et al., 1992). In contrast, the Vermicelli and Capellini Handling Tests extend from relatively recent characterizations of the adroit food handling behavior of rats (Whishaw and Coles, 1996). In both administration and analysis, these tests could undoubtedly be further refined and extended, e.g., to more precisely quantify fine digit and bimanual movement patterns and to quantify the coordinated movements of the tongue, mouth and jaw.

\section{Disclosures}

No conflicts of interest declared.

\section{Acknowledgements}

The authors would like to thank Dr. DeAnna Adkins, Dr. Marty Woodlee, Cole Husbands, Mónica A. Maldonado, Jackie Kane, and Dr. Tim Schallert for assistance in developing the Vermicelli Handling Test, and Lu Chou for assistance in developing the Capellini Handling Test. Supported by grants from the NIH-NINDS (NS05689) and NIH-NIA (1F31AG034032-01).

\section{References}

1. Allred, R.P., Adkins, D.L., Woodlee, M.T., Husbands, L.C., Maldonado M.A., Kane, J.R., Schallert, T. \& Jones, T.A. The Vermicelli Handling Test: a simple quantitative measure of dexterous forepaw function in rats. J. Neurosci. Methods 170, 229-244 (2008).

2. Baird, A.L., Meldrum A. \& Dunnett, S.B. The staircase test of skilled reaching in mice. Brain Res. Bull. 54, $243-250$ (2001). 
3. Ballermann, M., Metz, G.A., McKenna, J.E., Klassen, F. \& Whishaw, I.Q. The pasta matrix reaching task: a simple test for measuring skilled reaching distance, direction, and dexterity in rats. J. Neurosci. Methods 106, 39-45 (2001).

4. Castro, A.J. Motor performance in rats. The effects of pyramidal tract section. Brain Res. 44, 313-323 (1972).

5. Farr, T.D. \& Whishaw, I.Q. Quantitative and qualitative impairments in skilled reaching in the mouse (Mus musculus) after a focal motor cortex stroke. Stroke 33, 1869-1875 (2002).

6. Montoya, C.P., Campbell-Hope, L.J., Pemberton K.D. \& Dunnett, S.B. The staircase test : a measure of independent forelimb reaching and grasping abilities in rats. J. Neurosci. Methods 36, 219-228 (1991).

7. Peterson, G.M. Mechanism of handedness in the rat. Comp Psych Monographs 9, 1-67 (1934).

8. Tennant, K.A., Adkins, D.L., Donlan, N.A., Asay A.L., Thomas N., Kleim, J.A. \& Jones, T.A. The effect of age on motor skill learning and organization of the forelimb motor cortical representation in C57BL/6 mice. Society for Neuroscience Annual Meeting abstract. 783.4 (2009).

9. Tennant, K.A. \& Jones, T.A. Sensorimotor behavioral effects of endothelin-1 induced small cortical infarcts in C57BL/6 mice. J. Neurosci. Methods 181, 18-26 (2009).

10. Whishaw, I.Q. \& Coles, B.L. Varieties of paw and digit movement during spontaneous food handling in rats: postures, bimanual coordination, preferences, and the effect of forelimb cortex lesions. Behav. Brain Res. 77, 135-148 (1996).

11. Whishaw, I.Q. Coles, B.L., Pellis, S.M., \& Miklyaeva, E.I. Impairments and compensation in mouth and limb use in free feeding after unilateral dopamine depletions in a rat analog of human Parkinson s disease. Behav Brain Res. 84, 167-177 (1997).

12. Whishaw, I.Q., Dringenberg, H.C. \& Pellis, S.M. Spontaneous forelimb grasping in free feeding by rats: motor cortex aids limb and digit positioning. Behav. Brain Res. 48, 112-125 (1992).

13. Whishaw, I.Q., O Connor, W.T. \& Dunnett, S.B. The contributions of motor cortex, nigrostriatal dopamine and caudate-putamen to skilled forelimb use in the rat. Brain 109, 805-843 (1986). 